\title{
INTERMITTENT MAGNETIC FIELDS GENERATED BY TURBULENCE IN GALAXIES AND GALAXY CLUSTERS
}

\author{
D.D. SOKOLOFF \\ Physics Department, Moscow University, Moscow 119899, USSR \\ A.A. RUZMAIKIN and A. SHUKUROV \\ IZMIRAN, Troitsk, Moscow Region 142092, USSR
}

\begin{abstract}
The turbulence of intracluster gas in galaxy clusters and interstellar gas in galaxies can act as a dynamo generating chaotic magnetic fields. These fields are concentrated in ropes with the doubled radius of curvature of about the turbulent correlation length $l$ and thickness $l R_{\mathrm{m}}^{-1 / 2}$, where $R_{\mathrm{m}}$ is the magnetic Reynolds number. The field strength within the ropes is close to equipartition with turbulent kinetic energy. These results favorably agree with high-resolution observations of the galaxy cluster around Cyg A and correlation analysis of the Galactic nonthermal background. Ropy magnetic fields in interstellar gas lead to observable variations of, e.g., the Faraday depth at time scale of 1 month.
\end{abstract}

\section{Introduction}

Magnetic fields observed in galaxy clusters and in interstellar gas are very dissimilar. Apart from chaotic magnetic field, spiral galaxies possess a noticeable regular component of the field whereas only chaotic magnetic fields permeate intergalactic gas of some galaxy clusters. The theory of generation of regular magnetic fields is better known and developed, but the modern dynamo theory has made possible the investigation of chaotic magnetic fields in turbulent flows of conducting fluid (plasma). The most important necessary condition for excitation of the regular magnetic field is the overall rotation of a parent object which results in non-vanishing mean helicity of turbulence. Such rotation is typical of spiral galaxies where we evidence global magnetic structures which stand out on the background of (at least) equally strong chaotic interstellar fields. Galaxy clusters do not exhibit any considerable rotation. Thus, in accordance with the dynamo theory, in normal galaxy clusters only chaotic magnetic fields have been detected.

As shows the theory of generation of magnetic fields in non-rotating turbulent objects, the chaotic field grows in time if magnetic Reynolds number exceeds the critical value $R_{\mathrm{m}} \mathrm{cr} \simeq 50$. This condition, which is usually fulfilled in astrophysical plasmas, is the only prerequisite of the dynamo in a random flow. The characteristic time of exponential growth of the field at initial, kinematic stage of the dynamo is estimated as $l / v$, where $l$ and $v$ are the correlation scale and r.m.s. velocity of random flow, respectively. The modern theory of fluctuation dynamo is based on both analytical models which consider short-correlated velocity fields (see, e.g., Zeldovich et al., 1988, Ch. 5) and direct numerical simulations (e.g., Léorat et al., 1981). 
The fluctuation dynamo theory leads to an unexpected conclusion: even when the random velocity field has gaussian statistical properties, the generated random magnetic field is far from being gaussian. In this situation, evaluation of the correlation function or the energy spectrum is only the first step in both theory and observations because the higher is the order of a statistical moment of the magnetic field, the higher is its magnitude and the larger is its growth rate at the linear stage of the dynamo. In physical terms, this means that the generated field is concentrated within ropes. This property is called the spatial intermittency. It is important that ropiness of the generated magnetic field persists at nonlinear stage of the dynamo when back-action of the field on plasma motions leads to saturation of the exponential growth and development of the steady state. It seems plausible that high-resolution observations of magnetic fields in the galaxy cluster surrounding Cyg A (Dreher et al., 1987) reveal this ropy magnetic structure (Ruzmaikin et al., 1989).

\section{The Fluctuation Dynamo in Galaxy Clusters}

Inevitable presence in the intracluster gas of at least weak magnetic field of the strength $10^{-8} \mathrm{G}$ produced by remnants of radiogalaxies or galactic winds permits the MHD description of the intracluster plasma. Ruzmaikin et al. (1989) obtain the following estimates of turbulence parameters for the Coma cluster: $l \simeq 20 \mathrm{kpc}, v \simeq$ $400 \mathrm{~km} \mathrm{~s}^{-1}$ and $R_{\mathrm{m}} \simeq 8 \times 10^{7}$ with $R_{\mathrm{m}}$ the magnetic Reynolds number. It is presumed that the turbulence arises in galactic wakes.

For large $R_{\mathrm{m}}$ magnetic ropes of several different types can be excited. The first one is a simple magnetic rope whose doubled curvature radius and thickness are about $l$ and $l R_{\mathrm{m}}^{-1}-2$, respectively; the next mode is characterized by additional helical winding of the rope with diameter $l R_{\mathrm{m}}{ }^{-1 / 4}$, the third mode possesses two levels of helical windings with additional scale $l R_{\mathrm{m}}{ }^{-1} \gamma_{8}$, etc. The critical value of $R_{\mathrm{m}}$ for excitation of the $n^{\prime}$ th mode is about $\left(R_{\mathrm{m}} \mathrm{cr}\right)^{\mathrm{n}}$. Thus, for $R_{\mathrm{m}}=8 \times 10^{7}$ four or five such modes are excited. Correspondingly, the correlation function of magnetic field should show the following characteristic scales:

$$
20,6,2,0.2 \text {, and } 2 \times 10^{-3} \mathrm{kpc}
$$

for the Coma cluster. Growth time of the lowest mode can be estimated to be as short as $7 \times 10^{7}$ yr. The larger scales probably manifest themselves in observations of Dreher et al. (1987) which reveal strong gradients and reversals of magnetic field (numerical estimates should be corrected for slightly different parameters of turbulence in the Cyg A cluster which is not so rich as the Coma cluster).

The steady-state strength of magnetic field within the ropes is, probably, determined by equipartition with turbulent kinetic energy, $H \simeq\left(4 \pi \rho v^{2}\right)^{1-2} \simeq 10 \mu \mathrm{G}$ with $\rho$ $\simeq 3 \times 10^{-3} \mathrm{~cm}^{-3}$ the plasma density. However, the r.m.s. magnetic field is considerably smaller, $<\mathrm{H}^{2}>1^{-2} \simeq 2 \mu \mathrm{G}$, due to the ropiness (small filling factor).

About half of the ropes are not closed and extend over distances considerably exceeding $l$. This fact can be important for analysis of heat conduction in the intracluster gas.

Generation of chaotic ropy magnetic fields in galaxy clusters seems to be a rather widespread phenomenon. This implies that rarity of radiohaloes in galaxy clusters is associated with low efficiency of energetic particles production although other explanations also might be possible. 


\section{The Fluctuation Dynamo in Galaxies}

The processes of generation of ropy chaotic magnetic fields, which are similar to those discussed above, inevitably occur also in extended components of radiogalaxies and in interstellar gas of elliptic and spiral galaxies. In this respect, elliptic galaxies and radio lobes differ from galaxy clusters only quantitatively. Generation of chaotic magnetic fields in radiogalaxies has been considered by De Young (1980) who employed Fourier transformations and analyzed only energy spectra. For this reason, intermittency hardly can be revealed in his model.

The situation in spiral galaxies is somewhat different. Apart from fluctuation dynamo, one more source of chaotic magnetic fields is present there, namely tangling of the regular field by turbulence (see Ruzmaikin and Shukurov, 1982). In contrast to the ropes generated by the fluctuation dynamo, this component of interstellar chaotic magnetic field has generally the same statistical properties as the turbulence which produces them (e.g., the gaussian ones).

Here we estimate parameters of dynamo ropes in spiral galaxies as an example. The typical, averaged value of magnetic Reynolds number in interstellar gas, based on ambipolar diffusivity, is about $10^{6}$ (see Ruzmaikin et al., 1988). Correspondingly, we expect that the ropes of three or four lowest modes are excited. Their thickness is about $l R_{\mathrm{m}}{ }^{-1 / 2} \simeq 0.1 \mathrm{pc}$, additional helical windings of higher modes have diameters $l R_{\mathrm{m}}^{-1 / 4} \simeq 3 \mathrm{pc}$ and $l R_{\mathrm{m}}{ }^{-1}-8 \simeq 20 \mathrm{pc}$ (the scale $l R_{\mathrm{m}}{ }^{-1} 16 \simeq 40 \mathrm{pc}$ also can be present), the doubled curvature radius is $l \simeq 100 \mathrm{pc}$ (Kleeorin et al., 1986). The field strength within the ropes can reach $4.5 \mu \mathrm{G}$.

It is interesting that advection of the ropes by interstellar turbulent motions can be observed directly. Indeed, magnetic ropes generated by the dynamo are highly concentrated and, within the rope, the field is distributed along the radius $x$ as $H$ o $\exp \left[-x^{2} /\left(R R_{\mathrm{m}}^{-1}\right)\right]$. Although the characteristic width of this distribution is $l R_{\mathrm{m}}{ }^{-1 / 2}$, near the rope surface the field varies at the scale $H /(d H / d x) \propto l R_{\mathrm{m}}^{-1}$. When the ropes are advected at velocity $v \simeq 10 \mathrm{~km} \mathrm{~s}^{-1}$, this leads to variations of, e.g., the Faraday depth at the corresponding time scale $l_{R^{m}}^{-1} v^{1} \simeq 1$ month.

This short time scale makes possible to detect magnetic ropes in the Milky Way through monitoring of pulsars or extragalactic radio sources. On the other hand, polarized emission of many types of extragalactic radio sources exhibits variability at comparable time scales. It seems plausible that fluctuation dynamo plays an important role in radiogalaxies, quasars and accretion disks around black holes.

To conclude, we note that observations of brightness fluctuations of the Galactic nonthermal background performed by Dagkesamansky and Shutenkov (1987) reveal the correlation function which is very similar to that of the lowest ropy dynamo mode. In particular, the observed correlation function is negative for angular separation of 80 which corresponds to $140 \mathrm{pc}$ if the distance to emitting volume is $1 \mathrm{kpc}$. This anticorrelation scale corresponds to the diameter of closed magnetic ropes. More detailed observations can reveal traces of higher modes.

\section{References}

Dagkesamansky, R.D and Shutenkov, V.R. (1987) 'Brightness variations of the radio background and structure of Galactic magnetic field', Pis'ma Astron. Zh. 13, 182-190.

Dreher, J.W., Carilli, C.L. and Perley, R.A. (1987) 'The Faraday rotation of Cygnus A: magnetic fields in cluster gas', Astrophys. J. 316, 611-625. 
Kleeorin, N.I., Ruzmaikin, A.A. and Sokoloff, D.D. (1986) 'Correlation properties of self-exciting fluctuative magnetic fields', in Plasma Astrophysics, ESA SP-251, European Space Agency, Paris, pp. 557-561.

Léorat, J., Pouquet, A. and Frisch, U. (1981) 'Fully developed MHD turbulence near critical Reynolds number', J. Fluid. Mech. 104, 419-444.

Ruzmaikin, A.A. and Shukurov, A.M. (1982) 'Spectrum of the galactic magnetic fields', Astrophys. Space Sci. 82, 397-407.

Ruzmaikin, A.A., Shukurov, A.M. and Sokoloff, D.D. (1988) Magnetic Fields of Galaxies, Kluwer Akademic Publishers, Dordrecht.

Ruzmaikin, A., Sokoloff, D. and Shukurov, A. (1989) 'The dynamo origin of magnetic fields in galaxy clusters', Mon. Notic. Roy. Astron. Soc. (in press).

Zeldovich, Ya.B., Molchanov, S.A., Ruzmaikin, A.A. and Sokoloff, D.D. (1988) 'Intermittency, diffusion and generation in a nonstationary random medium', Sov. Sci. Rev. C, Math. Phys. 7, 1-110.

Young, D.S. de (1980) 'Turbulent generation of magnetic fields in extragalactic radio sources', Astrophys. J. 241, 81-97.

PUDRITZ: (1) How would you characterize the turbulence in galactic wakes (e.g. Gaussian, non-Gaussian, other?). (2) Could you test your rope-predictions by appropriate radio observations?

SOKOLOFF: (1) Of course, initially the wake-turbulent picture is nonGaussian, intermittent. But it is possible that in clusters with high number of galaxies interaction of wakes results in quasi-Gaussian turbulence. The question is not clear, but the magnetic field generation is not critically dependent on the structure of turbulence (cf. results of Frisch, Pouquet, Léorat et al.).

(2) Some such possibilities give us the results of Dreher et al. In detail it is discussed in our paper (Ruzmaikin et al., 1989, MNRAS, in press).

MOUSCHOVIAS: For a galaxy, your typical turbulent element had a size $\approx 10-20 \mathrm{kpc}$, magnetic field $\approx 2 \mu \mathrm{G}$, and density $\approx 10^{-3} \mathrm{~cm}^{-3}$. This gives an Alfvén speed $\approx 140 \mathrm{~km} / \mathrm{s}$ and, therefore, an Alfvén crossing time somewhat longer than $10^{8}$ years. Observations tell us that there is a large number of processes occurring over time scales smaller than $10^{8}$ years within the size of your typical turbulent element. Could you, please, explain how such a turbulent element would not be disrupted but would, instead, maintain its integrity?

SOKOLOFF: The lifetime of the turbulent cell can be shorter than $10^{8}$ years but the results do not depend on this parameter.

DOLGINOV: The intermittency is usually defined as a consequence of the nonlinearity of the stochatic process. The small scales can, of course, appear in the linear case but it is not an intermittency in common using this definition. Can you explain how you introduce the intermittency? 
SOKOLOFF: I think that the most direct and clear example of intermittency gives us a picture of linear instability (for details see Zeldovich et al., 1988, Sov. Sci. Rev. Math. Phys. 7, 1 (Harwood Acad. Press)). Nonlinearity is inessential for this kind of intermittency.

BIERMANN: In your picture, what determines the strength of the magnetic field inside the rope? Local equipartition or volume-averaged equipartition seem to be the two extreme possibilities.

SOKOLOFF: Local equipartition determines the strength of the magnetic field in the rope. The mean value of magnetic energy within a turbulent cell is smaller due to intermittent properties of the magnetic field.

KULSRUD: What is the source of helicity in the Coma cluster? Is it sporadic?

SOKOLOFF: In our approach we do not use the random helicity to describe the dynamo process which gives a purely chaotic magnetic field. of course, in any 3-dimensionally developed turbulent flow the local helicity $x \sim \vec{v} \operatorname{rot} \vec{v}$ generally is not zero but the mean value $\langle\vec{v}$ rot $\vec{v}\rangle$ can vanish.

HEILES: In the interstellar medium, each of the major radio loops is associated with an HI feature that is offset in angle from the radio continuum. This has been interpreted in terms of shocks but it is intriguing now to have another possibility.

SOKOLOFF: In principle, it is possible but this has not been analyzed in detail. 